\title{
Maize Resistance to Stem Borers can be modulated by Systemic Maize Responses to Long-term Stem Tunneling
}

\author{
Victor Rodriguez ${ }^{1}$, Pablo Velasco ${ }^{2}$, Ana $\mathrm{Cao}^{1}$, Rogelio Santiago ${ }^{3}$, Rosa Malvar ${ }^{1}$, and Ana \\ Butron $^{1}$ \\ ${ }^{1}$ Mision Biologica de Galicia (CSIC) \\ ${ }^{2}$ CSIC-Misión Biológica de Galicia \\ ${ }^{3}$ Universidad de Vigo
}

May 5, 2020

\begin{abstract}
Scarce attention has been paid to maize (Zea mays L.) resistance induced by corn borer damage although evidence shows that induced defenses have lower resource allocation costs than constitutive defenses. The maize responses to short and long-term attacks by the Mediterranean corn borer (MCB, Sesamia nionagrioides) have been previously studied but suggested differences between responses could be due to experimental differences. Therefore, in the current study, a direct comparison between short and long-term responses has been made. The objectives were (i) to determine changes in the level of antibiosis of the stems induced by feeding of Sesamia nonagrioides (Mediterranean Corn Borer; MCB) larvae for two days (short-term feeding) and nine days (long-term feeding), (ii) to characterize the metabolome of the stem short and long-term responses to borer attack and (iii) to look for metabolic pathways that could modulate plant resistance to MCB. Adjustment of plant performance under subsequent conspecific attack due to previous insect damage was genotype-dependent; defenses were progressively induced in the resistant inbred and constitutive defenses were broken down in the susceptible inbred. Results suggest that the different resistance of the two inbreds to stem tunneling by MCB could depend on their ability to stablish a systemic response.
\end{abstract}

\section{Introduction}

Stem tunneling by maize stem borers is an important constraint to achieve the potential yield of maize varieties across the world (Malvar, Butron, Ordas \& Santiago, 2008). The maize plant protect themselves from borer attack using constitutive and induced defenses but much more attention has focused on studying the first ones although evidence indicates that induced defenses have lower resource allocation costs than constitutive defenses (Beeghly, Coors \& Lee, 1997, Cardinal \& Lee, 2005, Howe \& Jander, 2008, Karban, Agrawal \& Mangel, 1997, Klenke, Russell \& Guthrie, 1986, Santiago, Butron, Arnason, Reid, Souto \& Malvar, 2006, Santiago, Butron, Revilla \& Malvar, 2011, Santiago, Souto, Monetti, Ordas, Ordas \& Malvar, 2006, Santiago, Souto, Sotelo, Butron \& Malvar, 2003). Indeed, maize stem feeding by borers significantly modify antibiosis against stem borer larvae, although antibiosis changes depend on the genotype and the duration of feeding (Cao, Butrón, Malvar, Figueroa-Garrido \& Santiago, 2019, Dafoe, Thomas, Shirk, Legaspi, Vaughan, Huffaker, Teal \& Schmelz, 2013). Dafoe et al. (Dafoe et al. , 2013) reported that growth of stem borer larvae was significantly higher when fed with stem tissues preconditioned by $48 \mathrm{~h}$ of larval tunneling compared to untreated stem tissues; while Cao et al. (Cao et al. , 2019) stated that the effect of long-term attack by borers on the antibiotic properties of corn stems is genotype-dependent.

Previous studies have already shown that feeding-induced changes in plant metabolites influence the behavior and performance of conspecifics and that influence depends on the time-lag of induction (Poelman, Broekgaarden, Van Loon \& Dicke, 2008, Santiago, Cao, Butron, Lopez-Malvar, Rodriguez, Sandoya \& Mal- 
var, 2017, Su, Chen, Mescher, Peng, Xie, Wang, Wu, Liu, Li, Wang \& Zhang, 2018, Wang, Bezemer, van der Putten \& Biere, 2015). According to that, several authors have pointed out to different defense mechanisms involved in the response to long-term feeding by insects compared to those implicated in the early response (Donze-Reiner, Palmer, Scully, Prochaska, Koch, Heng-Moss, Bradshaw, Twigg, Amundsen, Sattler \& Sarath, 2017, Gutsche, Heng-Moss, Sarath, Twigg, Xia, Lu \& Mornhinweg, 2009, Uawisetwathana, Graham, Kamolsukyunyong, Sukhaket, Klanchui, Toojinda, Vanavichit, Karoonuthaisiri \& Elliott, 2015); more specifically, previous studies also suggested important differences between maize stem responses to short and long-term feeding by stem borers (Dafoe, Huffaker, Vaughan, Duehl, Teal \& Schmelz, 2011, Rodriguez, Padilla, Malvar, Kallenbach, Santiago \& Butron, 2018, Rodriguez, Santiago, Malvar \& Butron, 2012). The early stem response to feeding by corn borers was characterized by the activation of signaling mechanisms mediated by phytohormones, whereas these molecules were marginally involved in the long-term response (Dafoe et al. , 2011, Rodriguez et al. , 2012). The stem long-term response was characterized by reorganization of the primary metabolism and a strong redox response mainly mediated by germin-like proteins to produce anti-nutritive and toxic compounds that reduce insect viability (Rodriguez et al. , 2018). However, no studies have simultaneously characterized short and long-term responses to stem attack by stem borers and the current work would be the first attempt to do it. A direct comparison of both responses using several genotypes under the same experimental conditions will shed light on real differences which cannot be disentangled from experimental differences when results from different experiments are compared.

As the components of the metabolome can be viewed as the end products of gene expression, un-targeted metabolomics would be a valuable tool to simultaneously monitor all biological processes operating in the plant response to herbivory and to discover bioactive compounds involved in plant resistance (Jansen, Allwood, Marsden-Edwards, van der Putten, Goodacre \& van Dam, 2009, Schauer \& Fernie, 2006, Sumner, Mendes \& Dixon, 2003). Indeed, metabolomics has been proved as a valuable tool to characterize plant responses to feeding by insects and to identify plant metabolites contributing to increased susceptibility or resistance (Agut, Gamir, Jaques \& Flors, 2015, Kang, Yue, Xia, Liu \& Zhang, 2019, Liu, Hao, Hu, Zhang, Wan, Zhu, Tang \& He, 2010, Marti, Erb, Boccard, Glauser, Doyen, Villard, Robert, Turlings, Rudaz \& Wolfender, 2013, Ponzio, Papazian, Albrectsen, Dicke \& Gols, 2017, Tzin, Hojo, Strickler, Bartsch, Archer, Ahern, Zhou, Christensen, Galis, Mueller \& Jander, 2017, Wang, Qu, Zhang, Hu, Tang \& Lu, 2016, Widarto, Van der Meijden, Lefeber, Erkelens, Kim, Choi \& Verpoorte, 2006). Herbivory could induce extensive changes to both general and specialized metabolism to prevent the allocation of energy and nutrients to herbivore fitness, rather than to plant fitness because plant metabolites involved in defense are not just toxic, repellent, and/or anti-nutritive molecules, but compounds that could attract enemies of herbivores, participate in nutrient transport and storage to make nutrients less accessible to the insect or involved in phenology shifts that grant herbivore avoidance (Schuman \& Baldwin, 2016).

The objectives were (i) to determine changes in the level of antibiosis of the stems induced by feeding of Sesamia nonagrioides(Mediterranean Corn Borer; MCB) larvae for two days (short-term feeding) and nine days (long-term feeding), (ii) to characterize the metabolome of the stem short and long-term responses to borer attack and (iii) to look for metabolic pathways that could modulate plant resistance to MCB

\section{Material and Methods}

\section{Plant materials and treatments applications}

Two different maize inbred lines (PB130 and EP42) were used because they were classified, at harvest, as partially resistant (PB130) and susceptible (EP42) to MCB attack based on the length of tunnels made by larvae in the stems; and differed for the early response to stem tunneling by MCB larvae (Butron, Malvar, Cartea, Ordas \& Velasco, 1999, Butron, Malvar, Velasco, Revilla \& Ordas, 1998, Rodriguez et al., 2012). Maize plants were individually grown in pots under greenhouse conditions. Three consecutive sowings of 10 seeds per sowing for each treatment-genotype combination were made; consecutive sowings were separated by 10 days to guarantee that, during the bioassay, MCB larvae were always fed with stems from plants around tasseling stage. In the earliest sowing and within each genotype, 10 plants were infested nine days before the establishment of the bioassay by placing two $2^{\text {nd }}$ instar larvae between the stalk and the sheath of basal 
leaves, 10 plants were infested 48 hours before and 10 plants were left untreated (control). Infested pots were protected with nets to avoid larval dispersion to non-infested pots. As stems to feed the larvae were renewed during the bioassay, treatments were also done as described in the second and third sowings to guarantee that stem portions given to the larvae came from plants around the tasseling stage pre-conditioned by larval feeding for 48 hours, nine days or non-conditioned (control) [Figure S1].

\section{Non-choice feeding bioassays}

To study the effect of short and long term attack, MCB larvae were fed with stems of plants infested 48 hours (short-term attack) or nine days (long-term attack) before bioassay establishment or non-infested plants. $1^{\text {st }}$ instar MCB larvae were initially weighted and individually placed in plastic tubes on $2 \mathrm{~cm}$ sections of the stem below the main ear. Larvae were previously fed on maize-based artificial diet and maintained at starvation for $24 \mathrm{~h}$, presenting weights of 1-3 mg at the beginning of the bioassay. Sixty larvae per treatment and genotype were set and maintained in a growth chamber under controlled conditions of temperature and humidity (22 ${ }^{\circ} \mathrm{C}, 80 \% \mathrm{RH}$ ) and a photoperiod of 16L:8D. When necessary, new fresh stem portions of plants infested 48 hours and 9 days before and control plants were provided to the bioassay larvae. Larval weights and data related to dead larvae were recorded at $7,11,14,18,22$, and 26 after bioassay establishment.

A repeated-measure analysis was performed to test differences for larval weights using the PROC GLIMMIX procedure of SAS software (SAS, 2008, Stroup, 2013). Initial larval weight was included as covariate, genotype and treatment were set as fixed factors, and a first-order autoregressive covariance structure (AR-1) was chosen in the within-subject correlation. Within each genotype, differences for larval weight of treatments were tested at each time using least square (LS) means adjusted by the initial larval weight. Additionally, linear and quadratic coefficients of regression of larval weight on time were obtained for each treatmentgenotype combination. Within each genotype, the comparison of the larval growth curves of treatments was performed by making orthogonal contrasts among treatment regression parameters (intercept, linear and quadratic components, respectively) ( $p$ [?] 0.05)(Littell, Milliken, Stroup, Wolfinger \& O, 2006). The PROC LIFETEST procedure of SAS software was used to test differences of larval survival among treatments applied to the same genotype using the Kaplan-Meier method (SAS, 2008). The death of larvae was the event of interest and the missing and alive larvae at the end of the bioassay were treated as censored data. The homogeneity of the survival distributions was tested using the Šidák multiple-comparison adjustment for log-rank test ( $p$ [?] 0.05).

\section{Metabolomics characterization of maize responses to MCB attack}

Metabolomics profile was obtained in five biological replicates per each genotype-treatment combination. A $3 \mathrm{~cm}$ portion at the bottom of the stem internode bellow the ear was taken from each plant. Frozen samples were lyophilized and ground to a fine powder using an electric mill. Extraction was performed using $50 \mathrm{mg}$ of this powder through $500 \mu \mathrm{L}$ of $80 \%$ aqueous methanol by sonication for $15 \mathrm{~min}$. Samples were centrifuged for $10 \mathrm{~min}$ in order to remove plant debris $(16000 \times \mathrm{g}$, at room temperature). Supernatant was filtered through a $0.20 \mu \mathrm{m}$ micropore PTFE membrane and placed in vials for further analysis. Five $\mu \mathrm{L}$ of each sample were injected into an ultra-high-performance liquid chromatography (UHPLC) system (Thermo Dionex Ultimate $3000 \mathrm{LC}$ ) connected to a QTOF detector (Bruker Compact) with a heated electrospray ionization (ESI) source. Chromatographic separation was performed in a Intensity Solo 2 C18 column $(2.1 \times 100 \mathrm{~mm} 1.7$ $\mu \mathrm{m}$ pore size) (Bruker Daltonics, Germany) using a binary gradient solvent mode consisting of $0.1 \%$ formic acid in water (solvent A) and acetonitrile (solvent B). The following gradient was used: $3 \% \mathrm{~B}(0-3 \mathrm{~min})$, from $3 \%$ to $25 \%$ B (3-10 min), from $25 \%$ to $80 \%$ B (10-18 min), from $80 \%$ to $100 \%$ B (18-22 min), and held at $100 \% \mathrm{~B}$ until $24 \mathrm{~min}$. The flow rate was established at $0.4 \mathrm{~mL}$ min-1 and column temperature was controlled at $35{ }^{\circ} \mathrm{C}$.

MS data were acquired using an acquisition rate of $2 \mathrm{~Hz}$ over the mass range of 50-1200 m/z. Both polarities (+-) of ESI mode were used under the following specific conditions: gas flow $9 \mathrm{~L}$ min-1; nebulizer pressure 2.6 bar; dry gas 9 L min-1; dry temperature 220 degC. Capillary and endplate offset were set to 4500 and $500 \mathrm{~V}$, respectively. To monitor the performance of data acquisition, the run sequence was started with three 
blanks (methanol, the solvent used in sample extraction) and a standard compound (triphenyl phosphate in positive ionization mode and chloramphenicol in negative ionization mode). Auto MS/MS fragmentation in pooled samples was performed in order to facilitate compound identification. For MS/MS analysis data was acquire using an acquisition rate of $8 \mathrm{~Hz}$ and precursor ions collected using an absolute threshold of 1500 counts and a cycle time of $1.0 \mathrm{~s}$.

The algorithm T-Rex 3D from the MetaboScape 4.0 software (Bruker Daltoniks, Germany) was used for peak alignment and detection. The generated dataset was imported into Metaboanalyst (Chong, Wishart \& Xia, 2019) to perform statistical analyses. In order to remove non-informative variables, data was filtered using the interquantile range filter (IQR). Moreover, Pareto variance scaling was used to remove the offsets and adjust the importance of high- and low-abundance ions to an equal level. The resulting three-dimensional matrix (peak indices, samples and variables) was further subjected to multivariate data analysis. Within each inbred, partial least squares discriminant analysis (PLS-DA) was carried out to investigate and visualize the pattern of metabolite changes between the control and each infestation treatment. This analysis was applied to obtain an overview of the complete dataset and discriminate those variables that are responsible for variation between groups (control vs 48-hours feeding to characterize the short-term response to MCB attack and control vs9-days feeding to characterize the long-term response). The PLS-DA model was evaluated through a cross-validation (R2 and Q2 parameters). The quality assessment (Q2) and R2 statistics provide a qualitative measure of consistency between the predicted and original data or, in other words, estimates the predictive ability of the model.

For each inbred and infestation treatment, features with a VIP (variable importance in projection) score $>2$ in the PLS-DA model (control vs infestation treatment) were selected and considered as the most influential features in that inbred response to MCB attack. In many studies, a VIP value $>2$ is a correct threshold for feature selection, but this cut-off depends on the number of variables used. For tentative identification, accurate mass data and isotopic pattern distributions for the precursor and product ions (if available) were studied and compared to the spectral data of reference compounds with a mass tolerance of 5 ppm. PubChem(Kim, Chen, Cheng, Gindulyte, He, He, Li, Shoemaker, Thiessen, Yu, Zaslavsky, Zhang \& Bolton, 2019), MassBank (Horai, Arita, Kanaya, Nihei, Ikeda, Suwa, Ojima, Tanaka, Tanaka, Aoshima, Oda, Kakazu, Kusano, Tohge, Matsuda, Sawada, Hirai, Nakanishi, Ikeda, Akimoto, Maoka, Takahashi, Ara, Sakurai, Suzuki, Shibata, Neumann, Iida, Funatsu, Matsuura, Soga, Taguchi, Saito \& Nishioka, 2010), KEGG(Kanehisa, Sato, Kawashima, Furumichi \& Tanabe, 2016), KNApSAcK (Afendi, Okada, Yamazaki, Hirai-Morita, Nakamura, Nakamura, Ikeda, Takahashi, Altaf-Ul-Amin, Darusman, Saito \& Kanaya, 2012), Metlin (Guijas, Montenegro-Burke, Domingo-Almenara, Palermo, Warth, Hermann, Koellensperger, Huan, Uritboonthai, Aisporna, Wolan, Spilker, Benton \& Siuzdak, 2018), and Chemspider (Ayers, 2012) databases were used.

\section{Results}

The analysis of variance for larval weight evolution during 26 days showed that all sources of variation [random (first-order autoregressive covariance structure of within-subject correlation among weights recorded on the same lava; $\left.\sigma^{2}=0.7539 \pm 0.0163\right)$ and fixed effects] were significant $(p<0.05)$ except treatments, but the interaction treatment $\mathrm{x}$ genotype was significant suggesting that maize genotypes would differentially respond to feeding treatments. At the end of the bioassay, larvae reared on stems from control susceptible inbred plants weighed less than larvae fed on the resistant ones (Fig. 1). Resistant plants pre-conditioned by 48 hours of feeding by MCB larvae decreased significantly larval weight compared to its control, while average weight of larvae fed with susceptible plants pre-conditioned by 48 hours feeding did not differ from its corresponding control larval weight (Fig. 1 and 2). On the other hand, susceptible plants exposed to feeding by larvae for a longer period (nine days) had opposite effects on larval weight to resistant plants and long-term attack to the resistant genotype caused stronger effect on the weight of larvae than short-term attack. In addition, induced responses by long-term attack made that the mortality of larvae fed on the resistant plants significantly higher than that of larvae fed on the susceptible ones although no differences for mortality between both inbreds were detected at control conditions (Table 1). 
Attending to the metabolomics approach, 4362 different features were detected among the different samples (Supplementary table S1). 194 and 192 ions were selected as important features (based on VIP-score values of the PLS-DA analysis) in the short and long-term responses, respectively, of the susceptible inbred line; 108 of them being important in both responses (Fig. 3). On the other hand, 101 ions out of the 188 and 227 detected as relevant in the short and long-term responses of the resistant inbred, respectively, were common in both responses. Although 194 and 188 ions had important contributions to the 48h responses to $S$. nonagrioides in the susceptible and resistant inbreds, respectively, only 57 of them contributed to both responses. Ninety ions were selected as important features based on VIP-score values of the PLS-DA analysis for the responses to 9-day stem borer attack in both inbreds. Finally, 30 ions were involved in the four responses. Identification and characterization of the metabolites from which the ions detected could be derived have been done with the ions with relevant effects on the long term response, either detected across genotypes or genotype-specific (Table S2).

\section{Discussion}

In the current study, constitutive antibiosis was higher in the susceptible than in the resistant inbred, but data collected on field resistance (always evaluated under artificial infestation) of these inbreds in previous studies have always shown better performance of the resistant inbred suggesting that induced responses could have an important role in resistance (Butron et al. , 1999, Ordas, Malvar, Santiago \& Butron, 2010). According to that, stems of the resistant inbred pre-conditioned by continuous damage of MCB larvae reduced larval weight compared to the control; meanwhile larval effectors or a general decrease of the plant metabolism provoked by insect damage seems to disrupt constitutive defenses of the susceptible inbred because stems from susceptible plants pre-conditioned for a long period increased larval weights compared to the susceptible control plants. Dafoe et al (Dafoe et al. , 2011, Dafoe et al. , 2013) already showed that 24-48h feeding by Ostrinia nubilalis, the European corn borer, could increase stem susceptibility of a single genotype. In opposition, induction by MCB attack in the resistant inbred had a detrimental effect on the weight of larvae agreeing with the increased antibiosis of maize leaves infested with Ostrinia furnacalis , the Asian corn borer, recently reported (Guo, Guo, He, Bai, Zhang, Zhao \& Wang, 2017, Guo, Qi, He, Wu, Bai, Zhang, Zhao \& Wang, 2019). Therefore, in the current study, alteration of plant performance under subsequent conspecific attack due to previous insect damage was genotype-dependent as it has been previously reported in other plant-insect interactions (Su et al. , 2018).

Long-term attack to the resistant genotype caused stronger effect on the weight of larvae than short-term attack suggesting that defenses induced by MCB could rather be progressively accumulated, since the initiation of damage, than punctually increased. We hypothesize that, after a prolonged period of insect damage, progressive accumulation of induced defensive metabolites would allow the resistant inbred to perform better than the susceptible inbred against insect attack as it has been reported in previous studies (Butron et al. , 1999, Cao et al. , 2019). However, after nine days of insect feeding, although continuous insect damage has broken down constitutive defenses of the susceptible inbred, accumulated defenses in the resistant would not still be enough to outperform antibiosis on larval growth of the susceptible inbred. Even so, long term induced responses made the mortality of larvae fed on the resistant plants significantly higher than that of larvae fed on the susceptible ones. Therefore, better field performance of the resistant inbred against MCB attack would greatly depend on the accumulation of induced defenses by MCB feeding, accumulation being higher as exposure to insect damage is prolonged; while higher constitutive resistance does not guarantee good performance under MCB infestation because continuous insect damage could disrupt constitutive defenses. These results suggest that the level of field resistance of the studied inbred line rather depends on induced changes by MCB attack than on constitutive defenses and those changes are determined by the duration of insect feeding. Continuous damage by insect feeding seems to contribute to increased susceptibility or resistance depending on the genotype and those genotype-dependent changes could be as the result of reconfiguration of metabolism in attacked plants (Schuman \& Baldwin, 2016).

Under the hypothesis that the level of field resistance rather depends on induced changes by MCB attack than on constitutive defenses and those changes are determined by the duration of insect feeding, ions impli- 
cated in the long term response could be important determinants of genotype induced resistance. Agreeing with other studies on induced metabolic plant changes by insect attack, the primary metabolism was downregulated in both maize genotypes by MCB 9-day feeding, including sugar, amino acid fatty acid and vitamin metabolism, as well as the tricarboxylic acid (TCA) or Krebs cycle which implies a disruption of energy production (Kang et al. , 2019, Liu et al. , 2010, Sabino, Tavares, Riffel, Li, Oliveira, Feres, Henrique, Oliveira, Correia, Nascimento, Hawkes, Santana, Holmes \& Bento, 2019, Wanget al. , 2016). According to a general downregulation of energy production and metabolism, intermediates or end-products of the shikimate pathway were repressed by MCB infestation in the susceptible inbred since decreases of tryptophan, phenylalanine and beta-tyrosine levels and those of several phenolic and indole-related compounds derived from them were registered in that inbred after 9-day feeding. However, some compounds synthesized through the shikimate pathway, such as tryptophan, or derived from the end products of the pathway, such as the indole-derived DIMBOA glucoside (antibiotic against $S$. nonagrioides larvae) and indol-acrylate (plant hormone) or the phenylpropanoids methyl-4-methoxy-3-nitrobenzoate (probable insecticide), 4-hydroxy-6methylcoumarin (biocide), sinapaldehyde (intermediate in lignin formation) or o-hydroxyhippurate [also known as salicylurate] (insect antifeedant), were up-regulated in the resistant inbred by MCB feeding (Cutler, Cutler, Wright \& Dawson, 2002, Feng, Chen \& Zhang, 2018, Ortego, Ruiz \& Castanera, 1998, Stuart, Brooks, Prescott \& Blackwell, 2000). In general, the levels of these compounds are higher in untreated stems of the susceptible compared to the resistant inbred and remain higher after 9-days of larvae feeding. In addition, a decrease of several nitrogen-containing compounds was observed in the susceptible inbred, while those compounds increased in the resistant inbred after 9-day feeding, but, again, levels were still higher in the susceptible. Therefore, the inbred susceptible at field conditions seemed to constitutively possess a resistance metabolic array that is broken down by continuous insect feeding, meanwhile the field resistant inbred, appeared to acquire induced resistance by channeling metabolism toward biosynthesis of defensive metabolites like the benzoxazinoid DIMBOA glucoside and methyl-4-methoxy-3-nitrobenzoate (Schuman \& Baldwin, 2016). As metabolomics analyses were made using undamaged stem sections, herbivore challenged plants of the resistant inbred would be able of mounting systemically active defense responses meanwhile susceptible plants could not (Howe \& Jander, 2008). Other authors have demonstrated that maize herbivory by Mythimna separata conferred resistance to the subsequently infested caterpillars through systemic changes of benzoxazinoids and probably other defensive metabolites (Malook, Qi, Hettenhausen, Xu, Zhang, Zhang, $\mathrm{Lu}$, Li, Wang \& Wu, 2019). Findings agreed with expectations because plant response to insect attack has been proved to be genotype-dependent, resulting in increased levels of phenylpropanoids, hydroxamic acids or/and nitrogen-containing secondary metabolites in resistant genotypes; meanwhile increased susceptibility after insect attack in other genotypes was associated to reduced levels of phenylpropanoids (Biyiklioglu, Alptekin, Akpinar, Varella, Hofland, Weaver, Bothner \& Budak, 2018, Kang et al. , 2019, Liu et al. , 2010, Suet al. , 2018).

Enzymatic or chemical oxygenation of free or membrane-esterified polyunsaturated fatty acids produces oxylipins and, among them, jasmonic acid has been highlighted as a key phytohormone in mediating maize resistance response to herbivory by insects (Borrego \& Kolomiets, 2016, Christensen, Huffaker, Kaplan, Sims, Ziemann, Doehlemann, Ji, Schmitz, Kolomiets, Alborn, Mori, Jander, Ni, Sartor, Byers, Abdo \& Schmelz, 2015). Jasmonic acid is a 13-LOX $\alpha$-linolenic acid-derived plant oxylipin, 13-HPOTrE[R], being the first intermediate in the synthesis of jasmonic acid. This phytohormone was upregulated and downregulated by $48 \mathrm{~h}$ feeding in resistant and susceptible inbreds, respectively. In parallel, 13-hydroxylinolenic acid (13-HOTrE), that resulted from reducing HPOTrE[R] and is not a jasmonic acid intermediate, was downregulated by $48 \mathrm{~h}$ feeding in the resistant inbred contributing to the precursor pool leading to cyclization and eventual synthesis of jasmonic acid (Farmer, Caldelari, Pearce, Walkersimmons \& Ryan, 1994). Epoxy - and hydroxy - derivatives of linoleic acid resulting from the peroxygenase pathway have been described as fungitoxic oxylipins and the metabolite 13-HPODE that belong to that oxylipin group was reduced by $48 \mathrm{~h}$ feeding in the resistant inbred and by 9-day feeding in both, meanwhile vernoleate diminished after 9-day feeding in both (Blee, 2002, Kato, Yamaguchi, Uyehara, Yokoyama, Namai \& Yamanaka, 1983, Tsitsigiannis, Kunze, Willis, Feussner \& Keller, 2005). Therefore, we speculate that maintenance of the upregulation of jasmonic acid precursors after $48 \mathrm{~h}$ feeding in the resistant inbred could play an important role in inducing 
systemic resistance meanwhile resistance was not induced in the susceptible inbred because precursors were downregulated. Production of jasmonic acid is required for a systemic response to herbivory (Bosch, Wright, Gershenzon, Wasternack, Hause, Schaller \& Stintzi, 2014). Systemic response to parasites involves transduction processes in which transient shifts of intracellular and apoplastic $\mathrm{pH}$ are essential: rapid alkalinization of the apoplast is combined with intracellular acidification, loss of $\mathrm{K}^{+}$, and influx of $\mathrm{Ca}^{2+}$ and followed by an oxidative burst and up-regulation of several pathways involved in defense (Viehweger, Dordschbal \& Roos, 2002). In that context, authors proposed lysophosphatidylcholines as good candidates for transducing the elicitor-triggered signal; lysophosphatidylcholines would be intracytoplasmic messengers that would connect the activation of a stress-responding enzyme of the plasma membrane (phospholipase $\mathrm{A}_{2}$ ) with the production of vacuolar proton fluxes. According with that idea, levels of lysophosphatidylcholines in the stems of the resistant inbred, augmented after feeding by MCB larvae; meanwhile levels of lysophosphatidylcholines in the stems of the susceptible inbred, that showed increased susceptibility upon feeding, diminished after feeding.

In the stems of the resistant inbred, organic acids malate and, especially, malonate (only detected in stems of PB130 pre-conditioned by 9-day feeding) increased after 9-day feeding. This can be consequence of a high redox level in the stem cells of the resistant inbred because under those conditions, the TCA cycle in mitochondria is transformed to a "non-cyclic" partial TCA cycle supplying citrate for the synthesis of 2-oxoglutarate, glutamate, and malonate (citrate valve), while malate is stored and participates in the redox balance (via malate valve) (Igamberdiev \& Eprintsev, 2016). In that scenario, the 3-Hydroxy-3Methylglutarate that is an "off-product" intermediate in the leucine degradation process and accumulated at high levels in the stem of the resistant inbred after continuous feeding by MCB larvae could be proposed as an important agent in causing and maintaining oxidative burst since this metabolite cause acute disruption of redox homeostasis in animal tissues (da Rosa, Seminotti, Amaral, Fernandes, Gasparotto, Moreira, Gelain, Wajner \& Leipnitz, 2013). Agreeing with the hypothesis of high oxidative stress in the resistant inbred after 9-day feeding by MCB larvae, this inbred line presents a high ROS scavenging level that increased after long-term feeding by MCB larvae because glutathione is higher in the resistant than in the susceptible inbred at control conditions and glutathione levels were doubled in both inbreds after 9-day damage by MCB larvae; in parallel, oxoproline that is a reservoir of glutamate and participates in glutathione homeostasis decreased in the susceptible but augmented in the resistant inbred (Ohkama-Ohtsu, Oikawa, Zhao, Xiang, Saito \& Oliver, 2008).

Longer maintenance of high jasmonic acid precursor levels and up-regulation of lysophosphatidylcholines could act as signal molecules of induced systemic resistance (ISR) to stem tunneling by stem borers. ISR would imply an oxidative burst (counterbalanced by increased ROS scavenging metabolites) and upregulation of metabolites involved in defense. Therefore, the different resistance of the two inbreds to stem tunneling by MCB larvae could depend on their ability to stablish that systemic response. Similarly, the observed differential defense responses of two different switchgrass cultivars to fall armyworm herbivory indicate that the resistant cultivar mounted a more robust response with potential activation of pathways that could lead to the production of antifeedants as compared to the susceptible (Palmer, Basu, Heng-Moss, Bradshaw, Sarath \& Louis, 2019).

We hypothesize that the level of field resistance rather depends on induced changes by MCB attack than on constitutive defenses and those changes are determined by the duration of insect feeding. Therefore, differential defense responses to continuous MCB feeding would result in resistance differences and ions that were differentially induced in both inbreds by long-term feeding could play an important role in resistance. A limit number of differentially induced features could be assigned to known metabolites but point to the ability of the inbred to establish a systemic response, involving oxidative burst and up-regulation of defense compounds, as determinant for limiting damage by MCB larvae.

\section{Acknowledgments}

This research was funded by the project RTI2018-096779 (MCIU/AEI/FEDER, UE). R. Santiago acknowledges postdoctoral contract "Ramón y Cajal" financed by the Ministry of Economy and Competiveness 
(Spain), Vigo University, and the European Social Fund. A. Cao acknowledges a contract as research-assistant technician (PTA2018-015873-I funded by MCIU/AEI/FEDER, UE)

\section{Conflict of interest statement}

I confirm that no authors have conflict of interest

\section{References}

Afendi F.M., Okada T., Yamazaki M., Hirai-Morita A., Nakamura Y., Nakamura K., Ikeda S., Takahashi H., Altaf-Ul-Amin M., Darusman L.K., Saito K. \& Kanaya S. (2012) KNApSAcK Family Databases: Integrated Metabolite-Plant Species Databases for Multifaceted Plant Research.Plant and Cell Physiology, 53 , 12.

Agut B., Gamir J., Jaques J.A. \& Flors V. (2015) Tetranychus urticae-triggered responses promote genotypedependent conspecific repellence or attractiveness in citrus. New Phytologist ,207 , 790-804.

Ayers M. (2012) ChemSpider: The Free Chemical Database. Reference Reviews , 26 , 45-46.

Beeghly H.H., Coors J.G. \& Lee M. (1997) Plant fiber composition and resistance to European corn borer in four maize populations.Maydica , 42, 297-303.

Biyiklioglu S., Alptekin B., Akpinar B.A., Varella A.C., Hofland M.L., Weaver D.K., Bothner B. \& Budak H. (2018) A large-scale multiomics analysis of wheat stem solidness and the wheat stem sawfly feeding response, and syntenic associations in barley, Brachypodium, and rice. Functional \& Integrative Genomics , $18,241-259$.

Blee E. (2002) Impact of phyto-oxylipins in plant defense. Trends in Plant Science, 7 , 315-321.

Borrego E.J. \& Kolomiets M.V. (2016) Synthesis and functions of jasmonates in maize. Plants-Basel , 5 .

Bosch M., Wright L.P., Gershenzon J., Wasternack C., Hause B., Schaller A. \& Stintzi A. (2014) Jasmonic acid and its precursor 12-Oxophytodienoic acid control different aspects of constitutive and induced herbivore defenses in tomato. Plant Physiology ,166 , 396-U574.

Butron A., Malvar R.A., Cartea M.E., Ordas A. \& Velasco P. (1999) Resistance of maize inbreds to pink stem borer. Crop Science ,39 , 102-107.

Butron A., Malvar R.A., Velasco P., Revilla P. \& Ordas A. (1998) Defense mechanisms of maize against pink stem borer. Crop Science, 38 , 1159-1163.

Cao A., Butrón A., Malvar R.A., Figueroa-Garrido D. \& Santiago R. (2019) Effect of long-term feeding by borers on the antibiotic properties of corn stems. Journal of Economic Entomology ,112 , 1439-1446.

Cardinal A.J. \& Lee M. (2005) Genetic relationships between resistance to stalk tunneling by the European corn borer and cell wall components in maize population B73xB52. Theoretical and Applied Genetics ,111, $1-7$.

Cutler H., Cutler S., Wright D. \& Dawson R. (2002) Method of controlling zoological and aquatic plant growth. Garnett, Inc., Watkinsville, GA (US) US.

Chong J., Wishart D.S. \& Xia J. (2019) Using MetaboAnalyst 4.0 for comprehensive and integrative metabolomics data analysis. Current Protocols in Bioinformatics , 68 , e86.

Christensen S.A., Huffaker A., Kaplan F., Sims J., Ziemann S., Doehlemann G., Ji L.X., Schmitz R.J., Kolomiets M.V., Alborn H.T., Mori N., Jander G., Ni X.Z., Sartor R.C., Byers S., Abdo Z. \& Schmelz E.A. (2015) Maize death acids, 9-lipoxygenase-derived cyclopente(a)nones, display activity as cytotoxic phytoalexins and transcriptional mediators. Proceedings of the National Academy of Sciences of the United States of America , $112,11407-11412$.

da Rosa M.S., Seminotti B., Amaral A.U., Fernandes C.G., Gasparotto J., Moreira J.C.F., Gelain D.P., Wajner M. \& Leipnitz G. (2013) Redox homeostasis is compromised in vivo by the metabolites accumulating 
in 3-hydroxy-3-methylglutaryl-CoA lyase deficiency in rat cerebral cortex and liver. Free Radical Research, $47,1066-1075$.

Dafoe N.J., Huffaker A., Vaughan M.M., Duehl A.J., Teal P.E. \& Schmelz E.A. (2011) Rapidly induced chemical defenses in maize stems and their effects on short-term growth of Ostrinia nubilalis . Journal of Chemical Ecology , 37, 984-991.

Dafoe N.J., Thomas J.D., Shirk P.D., Legaspi M.E., Vaughan M.M., Huffaker A., Teal P.E. \& Schmelz E.A. (2013) European corn borer (Ostrinia nubilalis ) induced responses enhance susceptibility in maize. Plos One , 8 .

Donze-Reiner T., Palmer N.A., Scully E.D., Prochaska T.J., Koch K.G., Heng-Moss T., Bradshaw J.D., Twigg P., Amundsen K., Sattler S.E. \& Sarath G. (2017) Transcriptional analysis of defense mechanisms in upland tetraploid switchgrass to greenbugs. BMC Plant Biology ,17 , 46.

Farmer E.E., Caldelari D., Pearce G., Walkersimmons K. \& Ryan C.A. (1994) Diethyldithiocarbamic acid inhibits the octadecanoid signaling pathway for the wound induction of proteinase-inhibitors in tomato leaves. Plant Physiology, $106,337-342$.

Feng Y., Chen J. \& Zhang A.J. (2018) Commercially available natural benzyl esters and their synthetic analogs exhibit different toxicities against insect pests. Scientific Reports , 8 .

Guijas C., Montenegro-Burke J.R., Domingo-Almenara X., Palermo A., Warth B., Hermann G., Koellensperger G., Huan T., Uritboonthai W., Aisporna A.E., Wolan D.W., Spilker M.E., Benton H.P. \& Siuzdak G. (2018) METLIN: A technology platform for identifying knowns and unknowns.Analytical Chemistry, 90 , 3156-3164.

Guo J.F., Guo J.Q., He K.L., Bai S.X., Zhang T.T., Zhao J.R. \& Wang Z.Y. (2017) Physiological responses induced by Ostrinia furnacalis (Lepidoptera: Crambidae) feeding in maize and their effects on O. furnacalis performance. Journal of Economic Entomology , 110 , 739-747.

Guo J.F., Qi J.F., He K.L., Wu J.Q., Bai S.X., Zhang T.T., Zhao J.R. \& Wang Z.Y. (2019) The Asian corn borer Ostrinia furnacalis feeding increases the direct and indirect defence of mid-whorl stage commercial maize in the field. Plant Biotechnology Journal , 17, 88-102.

Gutsche A., Heng-Moss T., Sarath G., Twigg P., Xia Y., Lu G. \& Mornhinweg D. (2009) Gene expression profiling of tolerant barley in response to Diuraphis noxia (Hemiptera: Aphididae) feeding.Bulletin of Entomological Research, 99 , 163-173.

Horai H., Arita M., Kanaya S., Nihei Y., Ikeda T., Suwa K., Ojima Y., Tanaka K., Tanaka S., Aoshima K., Oda Y., Kakazu Y., Kusano M., Tohge T., Matsuda F., Sawada Y., Hirai M.Y., Nakanishi H., Ikeda K., Akimoto N., Maoka T., Takahashi H., Ara T., Sakurai N., Suzuki H., Shibata D., Neumann S., Iida T., Funatsu K., Matsuura F., Soga T., Taguchi R., Saito K. \& Nishioka T. (2010) MassBank: a public repository for sharing mass spectral data for life sciences. Journal of Mass Spectrometry ,45 , 703-714.

Howe G.A. \& Jander G. (2008) Plant Immunity to Insect Herbivores.Annual Review of Plant Biology , 59 , 41-66.

Igamberdiev A.U. \& Eprintsev A.T. (2016) Organic acids: The pools of fixed carbon involved in redox regulation and energy balance in higher plants. Frontiers in Plant Science, $\mathbf{7}$.

Jansen J.J., Allwood J.W., Marsden-Edwards E., van der Putten W.H., Goodacre R. \& van Dam N.M. (2009) Metabolomic analysis of the interaction between plants and herbivores. Metabolomics ,5 , 150-161.

Kanehisa M., Sato Y., Kawashima M., Furumichi M. \& Tanabe M. (2016) KEGG as a reference resource for gene and protein annotation.Nucleic Acids Research , 44, D457-D462.

Kang K., Yue L., Xia X., Liu K. \& Zhang W.Q. (2019) Comparative metabolomics analysis of different resistant rice varieties in response to the brown planthopper Nilaparvata lugens Hemiptera: Delphacidae. 
Metabolomics, $\mathbf{1 5}, 13$.

Karban R., Agrawal A.A. \& Mangel M. (1997) The benefits of induced defenses against herbivores. Ecology , 78, 1351-1355.

Kato T., Yamaguchi Y., Uyehara T., Yokoyama T., Namai T. \& Yamanaka S. (1983) Self defensive substances in rice plant against rice blast disease. Tetrahedron Letters , 24, 4715-4718.

Kim S., Chen J., Cheng T.J., Gindulyte A., He J., He S.Q., Li Q.L., Shoemaker B.A., Thiessen P.A., Yu B., Zaslavsky L., Zhang J. \& Bolton E.E. (2019) PubChem 2019 update: improved access to chemical data.Nucleic Acids Research , 47 , D1102-D1109.

Klenke J.R., Russell W.A. \& Guthrie W.D. (1986) Recurrent selection for resistance to European corn-borer in a corn synthetic and correlated effects on agronomic traits. Crop Science, 26 , 864-868.

Littell R., Milliken G., Stroup W., Wolfinger R. \& O S. (2006)SAS for Mixed Models . (Second ed.). SAS Institute Inc, Cary, NC.

Liu C.X., Hao F.H., Hu J., Zhang W.L., Wan L.L., Zhu L.L., Tang H.R. \& He G.C. (2010) Revealing different systems responses to brown planthopper infestation for pest susceptible and resistant rice plants with the combined metabonomic and gene-expression analysis.Journal of Proteome Research , 9 , 6774-6785.

Malook S.U., Qi J.F., Hettenhausen C., Xu Y.X., Zhang C.P., Zhang J.X., Lu C.K., Li J., Wang L. \& Wu J.Q. (2019) The oriental armyworm (Mythimna separata) feeding induces systemic defence responses within and between maize leaves. Philosophical Transactions of the Royal Society B-Biological Sciences, 374, 12.

Malvar R.A., Butron A., Ordas B. \& Santiago R. (2008) Causes of natural resistance to stem borers in maize. In: Crop Protection Research Advances (ed E.N.B.a.P.V. Williams), pp. 57-100. Nova Science Publishers

Marti G., Erb M., Boccard J., Glauser G., Doyen G.R., Villard N., Robert C.A.M., Turlings T.C.J., Rudaz S. \& Wolfender J.L. (2013) Metabolomics reveals herbivore-induced metabolites of resistance and susceptibility in maize leaves and roots. Plant Cell and Environment ,36 , 621-639.

Ohkama-Ohtsu N., Oikawa A., Zhao P., Xiang C., Saito K. \& Oliver D.J. (2008) A gamma-glutamyl transpeptidase-independent pathway of glutathione catabolism to glutamate via 5-oxoproline in Arabidopsis . Plant Physiology , 148, 1603-1613.

Ordas B., Malvar R.A., Santiago R. \& Butron A. (2010) QTL mapping for Mediterranean corn borer resistance in European flint germplasm using recombinant inbred lines. BMC Genomics, 11 .

Ortego F., Ruiz M. \& Castanera P. (1998) Effect of DIMBOA on growth and digestive physiology of Sesamia nonagrioides (Lepidoptera : Noctuidae) larvae. Journal of Insect Physiology , 44, 95-101.

Palmer N.A., Basu S., Heng-Moss T., Bradshaw J.D., Sarath G. \& Louis J. (2019) Fall armyworm (Spodoptera frugiperda Smith) feeding elicits differential defense responses in upland and lowland switchgrass. Plos One , 14, 16 .

Poelman E.H., Broekgaarden C., Van Loon J.J.A. \& Dicke M. (2008) Early season herbivore differentially affects plant defence responses to subsequently colonizing herbivores and their abundance in the field.Molecular Ecology , 17, 3352-3365.

Ponzio C., Papazian S., Albrectsen B.R., Dicke M. \& Gols R. (2017) Dual herbivore attack and herbivore density affect metabolic profiles ofBrassica nigra leaves. Plant Cell and Environment ,40 , 1356-1367.

Rodriguez V.M., Padilla G., Malvar R.A., Kallenbach M., Santiago R. \& Butron A. (2018) Maize stem response to long-term attack bySesamia nonagrioides . Frontiers in Plant Science, $\mathbf{9}$.

Rodriguez V.M., Santiago R., Malvar R.A. \& Butron A. (2012) Inducible maize defense mechanisms against the corn borer Sesamia nonagrioides : A transcriptome and biochemical approach. Molecular Plant-Microbe Interactions , 25, 61-68. 
Sabino A.R., Tavares S.S., Riffel A., Li J.V., Oliveira D.J.A., Feres C., Henrique L., Oliveira J.S., Correia G.D.S., Nascimento T.G., Hawkes G., Santana A.E.G., Holmes E. \& Bento E.S. (2019) H-1 NMR metabolomic approach reveals chlorogenic acid as a response of sugarcane induced by exposure to Diatraea saccharalis . Industrial Crops and Products, $\mathbf{1 4 0}$.

Santiago R., Butron A., Arnason J.T., Reid L.M., Souto X.C. \& Malvar R.A. (2006) Putative role of pith cell wall phenylpropanoids inSesamia nonagrioides (Lepidoptera : Noctuidae) resistance. Journal of Agricultural and Food Chemistry, 54, 2274-2279.

Santiago R., Butron A., Revilla P. \& Malvar R.A. (2011) Is the basal area of maize internodes involved in borer resistance? BMC Plant Biology , 11, 12.

Santiago R., Cao A., Butron A., Lopez-Malvar A., Rodriguez V.M., Sandoya G.V. \& Malvar R.A. (2017) Defensive changes in maize leaves induced by feeding of Mediterranean corn borer larvae. BMC Plant Biology $, \mathbf{1 7}, 10$.

Santiago R., Souto X.C., Monetti L., Ordas B., Ordas A. \& Malvar R.A. (2006) Effect of maize pith free phenols on larval growth and development of Sesamia nonagrioides (Lepidoptera: Noctuidae). Journal of Entomology , 3, 281-289.

Santiago R., Souto X.C., Sotelo J., Butron A. \& Malvar R.A. (2003) Relationship between maize stem structural characteristics and resistance to pink stem borer (Lepidoptera : Noctuidae) attack. Journal of Economic Entomology , 96 , 1563-1570.

SAS (2008) SAS Institute Inc., Cary, NC, USA.

Schauer N. \& Fernie A.R. (2006) Plant metabolomics: towards biological function and mechanism. Trends in Plant Science, $\mathbf{1 1}, 508-516$.

Schuman M.C. \& Baldwin I.T. (2016) The layers of plant responses to insect herbivores. In: Annual Review of Entomology, Vol 61 (ed M.R. Berenbaum), pp. 373-394. Annual Reviews, Palo Alto.

Stroup W.W. (2013) Generalized linear mixed models: Modern Concepts, methods and applications . CRC Press, Boca Raton, Florida.

Stuart A.E., Brooks C.J.W., Prescott R.J. \& Blackwell A. (2000) Repellent and antifeedant activity of salicylic acid and related compounds against the biting midge, Culicoides impunctatus(Diptera : Ceratopogonidae). Journal of Medical Entomology ,37, 222-227.

Su Q., Chen G., Mescher M.C., Peng Z.K., Xie W., Wang S.L., Wu Q.J., Liu J., Li C.R., Wang W.K. \& Zhang Y.J. (2018) Whitefly aggregation on tomato is mediated by feeding-induced changes in plant metabolites that influence the behaviour and performance of conspecifics.Functional Ecology , 32 , 1180-1193.

Sumner L.W., Mendes P. \& Dixon R.A. (2003) Plant metabolomics: large-scale phytochemistry in the functional genomics era.Phytochemistry, 62, 817-836.

Tsitsigiannis D.I., Kunze S., Willis D.K., Feussner L. \& Keller N.P. (2005) Aspergillus infection inhibits the expression of peanut 13S-HPODE-forming seed lipoxygenases. Molecular Plant-Microbe Interactions , 18 , 1081-1089.

Tzin V., Hojo Y., Strickler S.R., Bartsch L.J., Archer C.M., Ahern K.R., Zhou S.Q., Christensen S.A., Galis I., Mueller L.A. \& Jander G. (2017) Rapid defense responses in maize leaves induced by Spodoptera exigua caterpillar feeding. Journal of Experimental Botany ,68 , 4709-4723.

Uawisetwathana U., Graham S.F., Kamolsukyunyong W., Sukhaket W., Klanchui A., Toojinda T., Vanavichit A., Karoonuthaisiri N. \& Elliott C.T. (2015) Quantitative H-1 NMR metabolome profiling of Thai Jasmine rice (Oryza sativa) reveals primary metabolic response during brown planthopper infestation. Metabolomics , $11,1640-1655$. 
Viehweger K., Dordschbal B. \& Roos W. (2002) Elicitor-activated phospholipase A(2) generates lysophosphatidylcholines that mobilize the vacuolar $\mathrm{H}+$ pool for $\mathrm{pH}$ signaling via the activation of Na+-dependent proton fluxes. Plant Cell , 14, 1509-1525.

Wang L.J., Qu L.J., Zhang L.W., Hu J.J., Tang F. \& Lu M.Z. (2016) Metabolic Responses of Poplar to Apripona germari (Hope) as Revealed by Metabolite Profiling. International Journal of Molecular Sciences $, 17,11$.

Wang M.G., Bezemer T.M., van der Putten W.H. \& Biere A. (2015) Effects of the Timing of herbivory on plant defense induction and insect performance in ribwort plantain (Plantago lanceolata L.) depend on plant mycorrhizal status. Journal of Chemical Ecology ,41 , 1006-1017.

Widarto H.T., Van der Meijden E., Lefeber A.W.M., Erkelens C., Kim H.K., Choi Y.H. \& Verpoorte R. (2006) Metabolomic differentiation ofBrassica rapa following herbivory by different insect instars using twodimensional nuclear magnetic resonance spectroscopy.Journal of Chemical Ecology , 32 , 2417-2428.

Table 1. MCB larval survival on non-choice feeding bioassay.

\begin{tabular}{|c|c|c|c|c|}
\hline Log-rank for treatments ${ }^{a}$ & Log-rank for treatments ${ }^{a}$ & Log-rank for treatments ${ }^{a}$ & Log-rank for treatments ${ }^{a}$ & Log-rank \\
\hline Treatment & Resistant $^{\mathrm{a}}$ & Susceptible & & Inbred \\
\hline Control & $0.14(27 \%) \mathrm{a}$ & $1.92(23 \%) \mathrm{a}$ & & Resistant \\
\hline $48 \mathrm{~h}$ & $-2.28(27 \%) \mathrm{a}$ & $1.05(22 \%) \mathrm{a}$ & & Susceptib \\
\hline $9 \mathrm{~d}$ & $2.01(33 \%) \mathrm{a}$ & $-2.97(17 \%) \mathrm{a}$ & & \\
\hline
\end{tabular}

Values of the log-rank statistic for testing homogeneity of survival distribution of MCB when larvae were reared on stems of plants infested with MCB larvae nine days (9d) or 48 hours (48h) before and on untreated (control) plants. Positive values of the log-rank statistic indicate that the number of dead larvae is over the number expected under the null hypotheses of equivalent survival distributions.

${ }^{a}$ Log-rank statistics followed by the same latter in the same column mean that survival curves of the different treatment within the same genotype were homogeneous $(p<0.05)$. The percentage shown between brackets is the mortality percentage.

${ }^{\mathrm{b}}$ Log-rank statistics followed by the same latter in the same column mean that survival curves were homogeneous between genotypes under the same treatment $(p<0.05)$.

\section{Figure legends}

Figure 1. Means of treatments applied to two inbreds for MCB larval weight at the end of bioassay. Mean comparison among treatments was separately made for each inbred (Capital letters for the resistant inbred and lower case letters for the susceptible inbred).

Figure 2. MCB larval growth on non-choice feeding bioassay. Regression curves (including lineal and/or quadratic coefficients) of the weight (mg) of the MCB larvae fed on stems from the resistant (A) and susceptible (B) inbred plants pre-infested with MCB larvae 48 hours (48h) or nine days (9d) before or untreated (control) on time. Treatments followed by different letters within the brackets [] showed regression curves significantly different based on differences between linear or/and quadratic coefficients.

Figure 3. Venn diagram showing the number of important features for each inbred response to each infestation treatment. Ions were considered as important for a particular response when their VIP-score values in the PLS-DA analysis were above 2.

\section{Supporting information}

Table S1. Areas for ions [named by retention time/ mass- to-charge ratio $(\mathrm{m} / \mathrm{z})$ ] detected in each inbredtreatment combination. Inbreds EP42 and PB130 are considered susceptible and resistant to attack by 
Sesamia nonagrioides, respectively. Treatments applied were: No treatment (Control), 9-day feeding bySesamia nonagrioides larvae (D9) and 2-day by Sesamia nonagrioides larvae (D2)

Table S2. Ions with a VIP (variable importance in projection) score $>2$ in the PLS-DA model for the long-term response (control vs 9-day infestation with MCB) in one inbred or in both inbreds (PB130 and EP42 considered as resistant and susceptible, respectively). The formula and name of metabolites identified as long as those PLS-DA models in which the feature shoes a VIP score $>2$ are also presented

Figure S1. Greenhouse experiment design, * maize samples for establishment of the bioassay and metabolite characterization were taken 77 days after sowing in the earliest planting. Maize tissues from the second and third plantings were taken at 84 and 91 days, respectively, to renew feeds for MCB larvae.

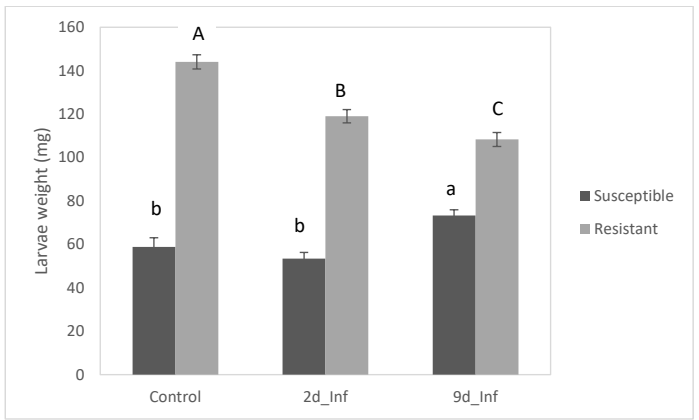


A

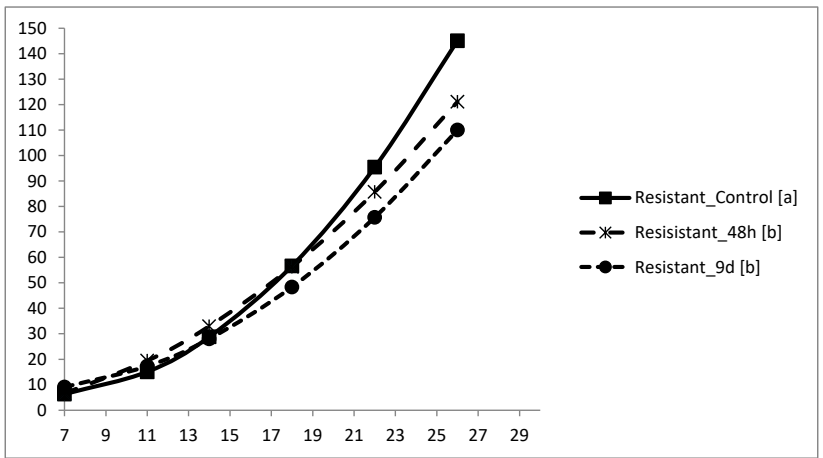

B

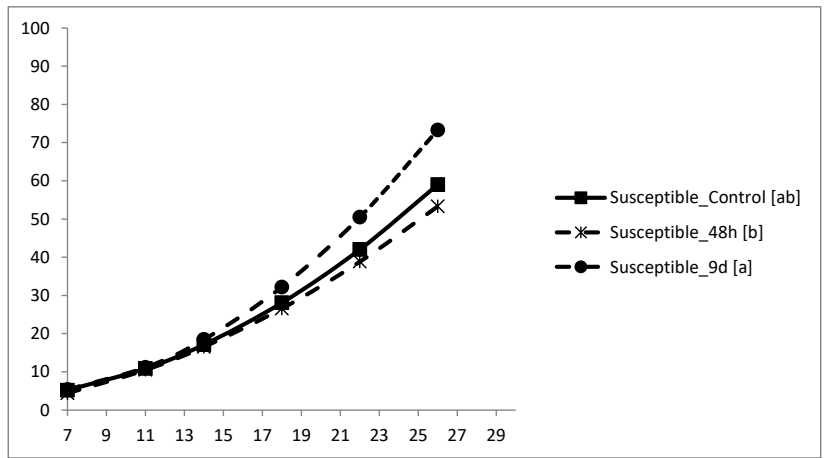

Hosted file

Figure 3.docx available at https://authorea.com/users/301720/articles/431589-maizeresistance-to-stem-borers-can-be-modulated-by-systemic-maize-responses-to-long-termstem-tunneling 